\title{
Prudente gobierno: LA PRUdencia EN EL GOBERNANTE*
}

\section{Prudent Government: the Prudence IN THE RULER}

\author{
María Montesinos Castañeda \\ Universitat de València
}

Recibido: 27/03/2019 Evaluado: 27/05/2019 Aprobado: 30/05/2019

Resumen: Partiendo de las fuentes filosóficas y literarias, la prudencia se considera la principal virtud que debe poseer todo gobernante. Esta relación propició la interacción visual entre la figura del gobernante y la imagen de la prudencia. De este modo, en ocasiones, esta cualidad adquiere atributos propios del gobernante, así como este adquiere los de la prudencia, retratándose incluso como esta misma virtud.

Palabras clave: prudencia, gobernante, iconografía, virtudes cardinales, cultura visual.

ABSTRACT: Beginning with the philosophical and literary sources, prudence is considered the main virtue that every ruler must possess. This relationship favoured the interaction between the figure of the ruler and prudence's image. In this way, prudence sometimes acquires the attributes of the ruler, just as he acquires those of prudence, portraying himself even as this virtue.

Keywords: Prudence, Ruler, Iconography, Cardinal Virtues, Visual Culture. 
T a prudencia -como una de las virtudes cardinales- ha sido definida, desaLrollada y dividida por numerosos pensadores a lo largo de la historia. De entre las reflexiones que suscita esta virtud podríamos distinguir tres tipos: aquellas que atienden a su significado, definición y funciones; las que versan sobre las subdivisiones de esta virtud en cuanto a las virtudes que la componen, y las que destacan su primacía entre las virtudes como la más propia de todo gobernante. Las reflexiones de la Antigüedad que destacan la prudencia como la principal virtud del gobernante, comenzaron a manifestarse visualmente a partir del medievo, adquiriendo una mayor importancia en la visualidad del gobernante a partir de la Edad Moderna. De este modo, veremos a continuación cómo el ejercicio gubernativo afectó a la imagen de la prudencia así como su visualidad se incorporó a las imágenes de gobernantes.

La prudencia, en resumen, consiste en saber deliberar, administrar los bienes, prever los acontecimientos y ser inteligente. ${ }^{1}$ A partir de estas consideraciones, desde la Antigüedad esta virtud ha estado asociada a la figura del gobernante como la cualidad principal que este debe poseer, por lo que Platón, en el segundo acto del Filebo, expone la importancia del poder ordenador del intelecto y la prudencia en el arte de gobernar. ${ }^{2}$ Por su parte, Aristóteles especificó más al respecto, distinguiendo incluso dos clases de prudencia política: «De la prudencia relativa a la ciudad, una, por así decirlo, arquitectónica, es legislativa, mientras que la otra, que está en relación con lo particular, tiene el nombre común de "prudencia política"». ${ }^{3}$ Estas consideraciones influyeron en el pensamiento posterior, siendo Platón ${ }^{4}$ y Aristóteles ${ }^{5}$ los referentes para los pensadores posteriores, quienes también la consideraron como propia del gobernante. Muestra de ello son las reflexiones de Cicerón ${ }^{6}$-quien consideró la prudencia la virtud por excelencia del hombre político ${ }^{7}$-, y Macrobio -quien, incluso, definió sus funciones en el gobierno- ${ }^{8}$ Sin embargo,

1. "Esta investigación se ha realizado gracias a la financiación de la Universitat de València y su programa de ayudas «Atracció de Talent».

Tomamos la definición de Aristóteles por ser muy específica y completa: «Corresponde a la sabiduría o prudencia el deliberar, el juzgar los bienes y los males y todas aquellas cosas que en la vida hay que desear o hay que evitar, le corresponde el emplear dignamente todos los bienes disponibles, el conducirse de forma recta en sociedad, el prestar atención a las ocasiones debidas, el utilizar el lenguaje y la acción de una manera sagaz e inteligente, el tener un conocimiento hondo de todas las cosas que son útiles». Arist. VV 4, 1250 a 30-40. Además, Aristóteles define la prudencia en más partes de su obra como, por ejemplo: Arist. $E N$ 6, 5 , 1140a 25; Arist. EN 6, 12, 1144a 5 y Arist. EN 6, 5, 1140b, entre otras.

2. Pl. Phlb. 2, 8, 28d-30a.

3. Arist. $E N$ 6, 8, 1141b 25. Aristóteles profundiza bastante en la cuestión especificando todas las vertientes que atañen a la considerada «prudencia política». Véase Arist. EN VI, 8, 1141b 20-30.

4. Pl. R. 6, 428d.

5. Arist. EN 6, 5, $1140 \mathrm{~b} 5$.

6. Véase Cic. rep. 2, 39; 2, 45; 2, 67; 4, 1; 4, 3; 6, 1.

7. Cic. rep. 6, 1. Véase Yves Lehmann: «Prudentia chez les penseurs romains. Essai d'investigation philosophique et morale», en Luigi De POLI (coor.): La Représentation de la Prudence, col. Chroniques Italiennes, Université de la Sorbonne Nouvelle-París III, París, 1999, pp. 13-14. Además, Cicerón consideró la prudencia como la primera de las cualidades que ha de poseer todo ciudadano. Véase CiC. rep. 5, 10.

8. MACR. somn. $1,8,7$. 
la aportación más relevante al respecto llegó siglos después de la mano de santo Tomás de Aquino. ${ }^{9}$

Ya en la Edad Media, las virtudes acompañaron a los diferentes gobernantes, tanto religiosos como civiles, en sus sepulcros ${ }^{10} \mathrm{y}$ espacios de trabajo (ayuntamientos, palacios, iglesias...), reforzando la honrada imagen de dichos personajes. ${ }^{11}$ Esta idea de la figura del gobernante como figura pública y la importancia de la tradición cristiana nos explican el valor que cobra la asociación de las virtudes con la persona, el mito y la imagen política de aquel. Por estos motivos, en los siglos siguientes tuvo lugar una notable interacción entre la imagen del gobernante y la prudencia, portando esta última atributos propios del gobierno, como son el cetro, la corona o, incluso, adquiriendo una imagen guerrera. Además, dichos aspectos visuales son consecuencia de las reflexiones de santo Tomás de Aquino, quien, dentro de las partes esenciales o especies de la prudencia en cuanto al Estado, distinguió la «prudencia gubernativa»y la «prudencia militar».

\section{Prudencia gubernativa}

La prudencia, al ser una virtud moral, implica un ámbito público y social, acerca de lo cual Santo Tomás explica que «la prudencia, orientada al bien común, se llama "prudencia política", de modo que hay la misma relación entre la prudencia pura y simplemente tal y la virtud moral que entre la prudencia política y la justicia legal», ${ }^{12}$ se trata de «la prudencia política, que versará acerca del bien común de la ciudad o de la nación». ${ }^{13}$. Por lo tanto, esta virtud se considera política y en relación con el gobernante por aplicarse a favor del bien común: «esta se llama prudencia en su razón común de prudencia, por ser recta dirección de las acciones, y es llamada política por su orden al bien común». ${ }^{14}$ De este modo, se estableció una asociación directa de esta con el acto de gobernar y, consecuentemente, con el gobernante y con el ciudadano. En consecuencia, la prudencia, al responder a su vertiente social, es parte del

9. Santo Tomás de Aquino define la prudencia: «La prudencia es, por tanto, sabiduría acerca de las cosas humanas: no sabiduría absoluta, por no versar sobre la causa altísima absoluta, puesto que trata del bien humano, y el hombre no es lo mejor de todo lo que existe. Por eso, con razón se dice allí que la prudencia es "sabiduría en el hombre", pero no la sabiduría en absoluto». S.Th. [40934] IIª-IIae q. 47 a. 2 ad 1.

10. Cabe recordar que, la presencia de las virtudes cardinales en los monumentos funerarios se remonta hasta el siglo xıIen Francia, aunque adquirió mayor popularidad a partir del siglo xıv en Italia, extendiéndose de nuevo por Francia y España en los siglos XV y xvI.

11. La idea de un "príncipe virtuoso» constituye la formulación en clave racional de las anteriores determinaciones de la figura del gobernante desde puntos de vista religiosos y civiles suponiendo la culminación del proceso idealizador de una imagen mítica del príncipe cristiano.

12. S.Th. [40998] II ${ }^{\mathrm{a}}$-IIae q. 47 a. 10 ad 1.

13. S.Th. [41005] II ${ }^{\mathrm{a}}$-IIae q. 47 a. 11 co.

14. S.Th. [41006] II ${ }^{\mathrm{a}}$-IIae q. 47 a. 11 ad 1. Además, Santo Tomás explicó más ampliamente dicha relación en S.Th. [41013] II ${ }^{\mathrm{a}}$-IIae q. 47 a. 12 co. 
bien común. ${ }^{15}$ Mediante estos argumentos y reflexiones que la relacionan con el gobernante, Santo Tomás llega a la conclusión de que: «La función principal del prudente es aconsejar bien». ${ }^{16}$ Con todo ello, la virtud de la prudencia es propia de cualquier ciudadano, pero sobre todo de cualquier gobernante, con el fin de guiarse bien en el camino de la vida tomando las decisiones correctas, fruto de una buena elección basada en la razón y a partir de las cuales se podrá dar un buen consejo. ${ }^{17}$

También en el pensamiento político de Alfonso X se puede encontrar uno de los ejemplos medievales más significativos del fuerte vínculo establecido entre el poder y la sabiduría, y este aspecto se puede analizar a partir de dos nociones distintas, aunque complementarias, de esta última. ${ }^{18}$ La primera comprende la sabiduría como capacidad de discernimiento entre lo «correcto» y lo «incorrecto», relacionándose con la virtud de la prudencia ${ }^{19}$ y con la propia función real del ejercicio político y de la administración de la justicia. A partir de esta concepción se formuló la idea de que un gobernante justo y sabio es aquel que prudentemente reflexiona sobre las decisiones que debe tomar, evitando actuar de modo impulsivo. ${ }^{20}$ Rucquoi explica claramente las funciones que permite la posesión de la sabiduría:

Saber de los saberes, la Sabiduría divina permite a quien la posee "ordenar los pueblos", "someter las naciones de las gentes", "ser rey y juez" y, además, conocer los secretos de la tierra y de los elementos, de los tiempos y de las estrellas y hasta de las cosas más ocultas y desconocidas de los hombres. ${ }^{21}$

Pero, en el Título V de la Segunda Partida, Alfonso X define, en primer lugar, las tres virtudes teologales seguidas de la función de las virtudes cardi-

15. «Ahora bien, siendo propio de la prudencia emitir los actos de consejo, deliberación o consejo, juicio e imperio respecto de los medios con los que se llega al fin debido, es claro que no solo se extiende al bien particular de un solo hombre, sino al bien común de la multitud». S.Th. [40997] IIª-IIae q. 47 a. 10 co.

16. S.Th. [41019] II'-IIae q. 47 a. 13 arg. 3.

17. En los Proverbios la misma sabiduría afirma dichas relaciones entre conceptos: «Yo, la Sabiduría, habito con la prudencia, yo he inventado la ciencia de la reflexión. [...] Míos son el consejo y la habilidad, yo soy la inteligencia, mía es la fuerza. Por mí los reyes reinan y los magistrados administran la justicia. Por mí los príncipes gobiernan y los magnates, todos los jueces justos». Pr 8,12,14-16.

18. MARINA Kleine: «La virtud de la prudencia y la sabiduría regia en el pensamiento político de Alfonso X el Sabio», en Res publica: revista de filosofía política, Universidad de Murcia, Murcia, 2007, p. 223.

19. Cabe mencionar que en la Antigüedad la prudencia y la sabiduría se empleaban como sinónimos, aunque Aristóteles especificó que la prudencia se diferencia de la sabiduría por ser una virtud activa en Arist, $E N$ VI, 5, 1140b 5. En el caso de Alfonso X, vemos cómo esta función selectiva de la prudencia se considera un tipo de sabiduría, tal y como lo había planteado Aristóteles. Dicha sinonimia se mantuvo en la época medieval, siendo incluso completado el elenco de las virtudes cardinales con la sabiduría ocupando el lugar de la prudencia. Santo Tomás explica esta relación en S.Th. [40934] IIª-IIae q. 47 a. 2 ad 1.

20. KLEINE: La virtud de la prudencia, p. 223.

21. Adeline Rucquoi: «De los reyes que no son taumaturgos: los fundamentos de la realeza en España», en Temas medievales, Programa de Investigaciones Medievales, Consejo Nacional de Investigaciones Científicas y Técnicas, Buenos Aires, 1995, p. 177. 
nales, empezando por la prudencia, a la que llama «cordura».22 Aunque identificada con una de sus partes,$^{23}$ la importancia de la prudencia -así como de las otras virtudes- en el ejercicio político propició la creación de numerosos «espejos de príncipes» a partir del siglo xiII es decir, de una literatura didáctica compuesta por los príncipes para su educación. ${ }^{24}$ Frère Laurent d'Orleans, por encargo del rey de Francia Felipe III (1245-1285) presentó, en el Somme le Roi, un pensamiento instructivo moral y religioso semejante al de Hugo de San Víctor. Sin embargo, al igual que Alfonso X, al enumerar las virtudes indicó que a «la premiere prudence» le corresponde la función de gobernar. ${ }^{25}$ Cabe destacar que la mayoría de los textos de los «espejos de príncipe», escritos por grandes retóricos, siguen la tradición aristotélica de una prudencia constituida por un saber experimental. ${ }^{26}$ Por su lado, Nicole Oresme (s. XIV), en su comentario de La política de Aristóteles, puso en evidencia que la prudencia es principal en la vida activa y práctica, considerando que la sabiduría no es suficiente para gobernar sino que es necesaria la prudencia debido a su ver-

22. «Cordura es la primera de las otras quatro virtudes, [...] que ha el Rey mucho menester, para biuir en este mundo bien derechamente. Ca esta faze ver las cosas, e judgarlas ciertamente, segund son, e pueden ser, e obrar en ellas, como deue, e non rebatosamente». Alfonso, Rey de Castilla: Las siete partidas del Rey Don Alfonso el Sabio, Benito Monfort, Valencia, 1767, fol. 4v. Disponible en Biblioteca Virtual de Pensamiento Político Hispánico Saavedra Fajardo. Partida II, Título v, Ley viII.

23. Los diferentes filósofos han considerado que la prudencia se divide en otras Virtudes (denominadas partes) que la componen. Véase Arist. $V V$ 4, 1250 a 30-40; Cic. inv. 2, 53, 160; Macr. somn. 1, 8, 7; Avg. octo quaest. 31; Hugo de san Víctor Summa Sententiarum, III (PL 176, 114); S.Th. [41055] II-IIae q. 48 co.; Bono Giamboni: Il libro de' vizî e delle virtudi e Il trattato di virtú e di vizî, G. Einaudi, Turín, 1968, p. 5; San Alberto Magno, Commentarii in librum Boethii “De Divisione”, tr. 4, c.1, pp. 74-75; Boeth. divis. (ML 64,888); FrAnCISCO DE IMPERIAL: El dezir a las syete virtudes y otros poemas, Espasa-Calpe, Madrid, 1977, pp. 107-112; JUAN DE MENA: «Coplas que fizo el famoso Juan de Mena contra los pecados mortales», en Raymond Foulché-Delbosch: Cancionero castellano del siglo XV, Bailly Bailliére, Madrid, 1912, p. 147; Francisco de Guzmán: Triumphos morales, Casa de Andrés, Alcalá de Henares, 1565, fol. 70r. Si agrupamos las diferentes divisiones consideradas obtenemos que las partes de la prudencia son: memoria, providencia/previsión, inteligencia, intelecto, razón, circunspección, docilidad, precaución/cautela, consejo, experiencia, temor de Dios, sagacidad, deliberación, elección, entendimiento, conocimiento y buena medida.

24. Myriam Chopin-Pagotto: «La prudence dans les Miroirs du prince», en Luigi de Poli (coor.): La Représentation de la Prudence, col. Chroniques Italiennes, Université de la Sorbonne Nouvelle-París III, París, 1999, p. 87.

25. «Esta prudencia tiene tres oficios. Para esta virtud todo lo que el hombre hace, dice o piensa, todo lo que él ordena, dirige y gobierna, es para conseguir la repercusión más correcta». Frère LAURENT: Le somme le roi, Paillarr, París, 2007, pp. 233-234.

26. Nathalie Dauvois: «Prudence et politique chez les grands rhétoriqueurs: Janus Bifrons», en Evelyne Berriot-Salvatore, Catherine Pascal y François Roudaut (dir.), La vertu de prudence: entre Moyen Âge et âge classique, Classiques Garnier, París, 2012, p. 56. Sin embargo, fue Petrus de Alvernia quien marcó la diferencia en esta concepción política de la prudencia, ya que solo la concibe como la virtud del buen hombre y nunca la del ciudadano. Véase. MArco Toste: «Virtue and the City: The Virtues of the Ruler and the Citizen in the Medieval Reception of Aristotle's Politics», en István P. BejCZy y CARY J. NeDerman (eds.), Princely virtues in the Middle Ages, 1200-1500, Brepols, Turnhout, Abingdon, 2007, p. 89. Parece que Petrus de Alvernia entendía que la virtud del buen ciudadano es un tipo de prudencia, ya que los actos del ciudadano son actos prudentes, pero no la llama prudencia en sí, reservando dicha denominación para el buen hombre. Véase MICHIEL VERwEIJ: «Princely Virtues or Virtues for Princes?», en IsTVÁN P. Bejczy y Cary J. Nederman (eds.), Princely virtues in the Middle Ages, 1200-1500, Brepols, Turnhout, Abingdon, 2007, p. 69. 
tiente práctica y política. ${ }^{27}$ Sin embargo, la sustitución de esta por la sabiduría fue predominante en muchos casos, como en la Ética de Aristóteles, atribuida al maestro de la coronación de Carlos VI. En esta obra, la prudencia es la única del grupo particularmente asociada con las calificaciones y conducta del gobernante ideal, pero desde Carlos VI fue conocida como sabiduría, aunque a su vez se identificaba con la representación de la prudencia. ${ }^{28}$ La conjunción del uso de la prudencia y la sabiduría, y su importancia en el gobierno, tienen lugar en el Epitre d'Othea de Cristina de Pizan (1364-1430), ${ }^{29}$ donde se expone el arquetipo de soberano ideal evocando a la prudencia como la cualidad esencial del buen gobernante. Dentro de este discurso ideológico, encontramos una fuerte influencia del pensamiento aristotélico proveniente de sus Éticas. ${ }^{30}$ Incluso dentro de la obra de Pizán se explica dicha influencia de la Antigüedad: «En nuestro tiempo, nosotros los cristianos, alumbrados por la gracia divina, somos capaces de restaurar a la moral las opiniones de los antiguos, y con este propósito, pueden componerse unas excelentes alegorías». ${ }^{31}$ La protagonista de la obra es Otea, la cual se define así: «Por "Othea" entendemos la virtud de prudencia y de sabiduría, que le adornaban. Y como las cuatro virtudes cardinales son necesarias para el buen gobierno, lo discutiremos en las siguientes líneas». ${ }^{32} \mathrm{~A}$ partir de estas premisas, se desarrolla una histórica alegórica entorno a las virtudes que representa a la diosa en relación con el ejercicio del gobierno: «Como la prudencia y la sabiduría son madre y arquitecto de todas las virtudes, sin las cuales no se puede gobernar a las demás, es necesario que la prudencia adorne el espíritu caballeresco».33

Todo este pensamiento en torno al gobernante y sus cualidades también quedó patente en las narraciones en las que las mismas virtudes intervienen. Este es el caso del Cancionero de Baena -donde se recogen una serie de composiciones dedicadas al nacimiento de Juan II en Toro el 6 de marzo de 1405y El dezir a las syete virtudes de Francisco Imperial -en el que se expone cómo al dar a luz la reina ante ella se aparecen ocho doncellas que representan las virtudes-. En la obra de Imperial, el cosmos se presenta a saludar al recién nacido futuro monarca, junto a los planetas, cada uno de ellos acompañado por una virtud. En primer lugar, Saturno comienza indicándole una serie de modelos clásicos de conducta y programas de grandeza, entregándole con ello la

27. Chopin-Pagotto: La prudence, p. 93.

28. Claire Richter: Imaging Aristotle: verbal and visual representation in fourteenth-century France, University of California Press, Berkeley, 1995, p. 119.

29. Existen precedentes carolingios de este género literario como De institutione regia de Jonas d'Orléans (s. Ix) y De regis persona et Regio ministerio de Hinemar de Reims. Véase Chopin-Pagotto: La prudence, p. 88.

30. Chopin-Pagotto: La prudence, pp. 87-89.

31. CRistina de PizÁn: La rosa y el príncipe: voz poética y voz política en las «Epístolas», Gredos, Madrid, 2005, p. 98.

32. Ibid.

33. PizÁn: La rosa y el príncipe, p. 100. 
prudencia: «E dóle a prudencia, esta mi doncella, / por su Mayordoma mayor, e con ella / será sin dubda mejor obrador». ${ }^{34}$ También Fray Íñigo de Mendoza, en su Vita Christi, describió la prudencia como la virtud que debe acompañar al gobernante:

Quien con esta maestría / bien desuia / lo sano de lo doliente, / meresçe, pues es prudente, / ciertamente / que tenga renta por guía; / de quien rige poliçia / yo diría / ques la prudencia su espejo, / por lo qual los del conçejo / al tiempo viejo / ordeno por compañía / ala real señoría. ${ }^{35}$

Es imprescindible que en los «espejos de príncipes» que continúan en estos siglos, se mencione la necesidad de la prudencia, por lo que Erasmo de Rotterdam escribió, en su Educación de un príncipe cristiano (1516), que entiende como «virtudes del príncipe» los atributos propios de un gran legislador, pero situado en un pueblo ordenado, pacífico, donde reina la prudencia, ${ }^{36}$ ya que la posesión y el gobierno de estas virtudes garantizará la estabilidad del pueblo. Por otra parte, Maquiavelo diseñó un «príncipe» con virtudes apropiadas para una situación excepcional, ${ }^{37}$ entre las cuales incluye de manera preeminente la prudencia: «es necesario a lo menos que tenga el príncipe bastante prudencia para preservarse de aquellos vicios y defectos que pudieran perderle», ${ }^{38}$ pues las virtudes maquiavelianas son básicamente las de los clásicos, las virtudes antiguas: poder, magnificencia, prudencia, austeridad, vigor y disciplina. Además, considera que «los fundadores del estado mezclan clemencia y crueldad, vigor y generosidad, prudencia y audacia». ${ }^{39}$ Del mismo modo, Maquiavelo puso en relación la prudencia con el consejo -una de sus partes-, por lo que explica: «de donde concluyo: que los consejos buenos, vengan de donde vengan, no son debidos sino a la prudencia del Príncipe, y que la prudencia del Príncipe no es sino el fruto de los buenos consejos ${ }^{40}$. No obstante, añade que esta virtud no puede aparentarse, pues solo los príncipes sabios pueden recibir sabios consejos. Es decir, que rodearse de buenos ministros, escucharlos y aceptar sus buenos consejos no es un medio de cubrir las deficiencias de un príncipe, sino la mejor prueba de la sabiduría y virtù de este. ${ }^{41}$ Asimismo,

34. Citado por Julio Vélez-SAinz: «La iconización de lo femenino en la Edad Media (de Prudencio a la corte de Juan II)», en Tejuelo: Didáctica de la Lengua y la Literatura, 2008, pp. 67-68.

35. Fray Íñigo de Mendoza: «Vita Christi techo por coplas por frey Yñigo de Mendoca a petiçion de la muy virtuosa señora doña Juana de Cartagena», en RAymond Foulché-Delbosch: Cancionero castellano del siglo XV, Bailly Bailliére, Madrid, 1912, p. 78.

36. José Manuel Bermudo: Maquiavelo, consejero de príncipes, Universitat de Barcelona, Barcelona, 1994, p. 82.

37. Bermudo: Maquiavelo, p. 82.

38. Nicolás Maquiavelo: El príncipe (Comentado por Napoleón Bonaparte), Santiago Rueda, Buenos Aires, 1968, p. 168.

39. Bermudo: Maquiavelo, pp. 90-95.

40. Maquiavelo: El príncipe (comentado), p. 198.

41. Bermudo: Maquiavelo, p. 241. 
para Maquiavelo es necesario por encima de todo que el rey sea prudente para evitar que lo acusen de los vicios que puedan arrebatarle el Estado. ${ }^{42}$ Aunque Maquiavelo defiende que existen cicios que en realidad son buenos y virtudes que se convierten en vicios, ahí es donde se produce el paso de la concepción de virtud medieval a la concepción maquiavélica de la virtù política. ${ }^{43}$ Podemos decir que la prudencia será una de las cualidades que más se tengan en cuenta para un soberano a lo largo de la época moderna, ya que este debe ser capaz de actuar con reflexión y precaución.

Por lo tanto, la prudencia será la encargada de dirigir el resto de virtudes y la propia del gobernante por excelencia, ${ }^{44}$ motivo por el cual en El gran carro triunfal de Maximiliano I, la alegoría que dirige a uno de los caballos que se posicionan en primer lugar es la experiencia (virtud base de la prudencia), ya que gracias a su bagaje es la más indicada para conducir al monarca, al contar con práctica suficiente para no incurrir en los mismos errores que en un pasado pudo cometer. Estas serán las claves de la nueva construcción en la que primó el saber actuar con cautela, calma y tranquilidad, pero a la vez de manera audaz y rápida cuando fuese necesario para solucionar los problemas. Por su parte, Sepúlveda (1490-1573), en De regno, expresa que quien desempeñaba el «oficio» de gobernante se entendía firmemente asentado sobre la prudencia, considerada como «facultad civil». ${ }^{45}$ Además, sobre esta prudencia puramente «política» se sobreponía otra que constituía el «hábito verdadero». ${ }^{46}$ Atendiendo a estas reflexiones, en las bodas imperiales de Carlos V (1526, Sevilla) se alzaron siete arcos triunfales dedicados a las virtudes, el primero de los cuales se dedicó a la prudencia, ${ }^{47}$ mientras que del segundo se describió: «El segundo arco estaba dedicado a la fortaleza, enseñando que después de la prudencia tiene el segundo lugar en los príncipes la fortaleza». ${ }^{48}$ De este modo, la fortaleza quedaba subordinada a la prudencia, la primera de las virtudes, enfatizando así la dependencia existente entre las virtudes cardinales con el fin de comple-

42. Nicolás Maquiavelo: El príncipe, Edaf, Madrid, 2009, pp. 147-150

43. Maquiavelo modifica la definición y objetivo de la prudencia establecidos anteriormente: «La prudencia humana sirve únicamente para escoger el menos perjudicial de los males conocidos». MAQUIAVELO: El príncipe, p. 138

44. Jorge Norberto Ferro: «Las virtudes del gobernante en las cuatro crónicas que preceden a la obra del canciller Ayala», en Anales de la Fundación Francisco Elías de Tejada, Fundación Francisco Elías de Tejada, Madrid, 1995, 1, p. 50. Aristóteles considera que la experiencia es esencial para la prudencia por lo que los jóvenes, al carecer de la primera, no pueden poseer esta virtud. Véase Arist. EN VI, 9, $1142 \mathrm{a} 15$.

45. PAblo Fernández: «Espejo de prudencia», en Francisco J. Rocha (coor.): La monarquía hispánica Felipe II, un monarca y su época: Real Monasterio de San Lorenzo del Escorial, 1 de junio, 10 de octubre, 1998, Sociedad Estatal para la Conmemoración de los Centenarios de Felipe II y Carlos V, Madrid, 1998, p. 76.

46. Fernández: Espejo de prudència, p. 76.

47. A. J. Morales: «Recibimiento y boda de Carlos V en Sevilla», en vv. AA.: La fiesta en la Europa de Carlos V: [exposición] Real Alcázar, Sevilla, 19 de septiembre-26 de noviembre 2000, Sociedad Estatal para la Conmemoración de los Centenarios de Felipe II y Carlos V, Madrid, 2000, p. 38.

48. Citado por JuAn CARMona: Iconografía clásica: guía básica para estudiantes, Istmo, Madrid, 2002, p. 247. 
tar el sentido propio de cada una. Por lo tanto, la prudencia era colocada en primer lugar como la «reina de todas las virtudes», idea que compartieron los tratadistas políticos del siglo XVI. ${ }^{49}$ Ante dicha asociación de la prudencia a la figura del gobernante en vida, no era de extrañar que también se estableciera tras la muerte de este. Por esta razón, en el túmulo que se erigió en los funerales de Carlos V en Valladolid (1559) encontramos que «los pedestales del cuadrado, con las colunas, eran dedicados a las quatro virtudes cardinales», las cuales se relacionan con los hechos heroicos de su carrera militar y política. La prudencia se une a unos cuadros que «representavan aquella prudencia y sciencia militar, de que usó el Emperador con tal celeridad qual nunca tuvo Iulio César, en ayuntar tan poderoso exército [...] con el qual hizo retirar al gran Turco que venía sobre Viena», siendo continuada por las otras virtudes, a las que siempre anteceden. ${ }^{50}$ Según Calvete de Estrella, la prudencia, así como las otras virtudes contribuían a definir más nítidamente las características de ese auténtico caballero cristiano. ${ }^{51}$ En esas escenas del túmulo se nos enseña cómo, a través del ejercicio de la virtud, el príncipe es capaz de construir la historia y alcanzar la fama, lo que le permite vencer a la muerte. Pero además de acompañarse de las virtudes, los gobernantes se retrataron como personificaciones de estas. Por este motivo, Luisa de Saboya (1476-1531) se hizo retratar acompañada de un gran compás y un ciervo, emblemas de la mesura ${ }^{52}$ y prudencia ${ }^{53}$ de sus actos (ca. 1510, París, BNF, ms. 12247) (fig. 1). Igualmente, la reina Elizabeth fue retratada sosteniendo un cedazo (atribuido a George Gower, 1579, Colección privada) (fig. 2), signo del discernimiento ${ }^{54}$ que caracteriza a la prudencia. ${ }^{55}$ Por lo tanto, la preocupación de los gobernantes por lo simbólico denotaba su interés por ser caracterizados por todas esas virtudes a las que se asocian, dando así una imagen de buen gobernante cristiano al ser poseedor de tales cualidades.

\footnotetext{
49. Fernando Checa: Carlos V y la imagen del héroe del Renacimiento, Taurus, Madrid, 1987, p. 177.

50. CheCA: Carlos V, p. 268.

51. AA. vv. La fiesta, p. 354.

52. «El compás significa orden y medida». CESARE RIPA: Iconología, Akal, Madrid, 2007, vol. 2, p. 182.

53. Ripa considera que el ciervo es un animal prudente porque «cuando sus largas y bien dispuestas patas le incitan a la carrera, prudentemente retrasa el grave peso de sus cuernos, así como el peligro de enredarse con ellos entre los bosques y malezas». Igualmente, Ripa considera que «Sirve también a nuestro propósito el rumiar de este animal, asemejándose al discurso y reflexión que preceden a la resolución de las buenas decisiones», características propias de la prudencia. RipA: Iconología, vol. 2, p. 236.

54. El cedazo es atributo de la prudencia durante los siglos XV y XVI en el ámbito de la «nueva visualidad» - propia de la visualidad francesa, aunque se extendió a otros territorios-. Ripa recoge este atributo como propio de la Sabiduría explicando: «otros la representaban con una criba o cedazo, mostrándose con ello aquel efecto de la sabiduría que consiste en distinguir y separar el grano de la paja, o también la buena simiente de la que es mala y perniciosa para las costumbres y acciones de los hombres». Ripa: Iconología, vol. 2, p. 282. Sin embargo, cabe tener en cuenta que desde la Antigüedad y a lo largo del medievo la sabiduría y la prudencia se emplearon como términos sinónimos, lo que repercutió en la imagen de ambas. Véase Somme le roi: 1295, París, BMaz, ms. 870-1, fol. 83v; y 1294 París, BNF, Français 938, fol. 69.

55. La reina Elizabeth I se retrató como alegoría de diferentes virtudes en muchas ocasiones. Véase Samuel C. Chew: The virtues reconciled: an iconographic study, University of Toronto Press, Toronto,
} 1947, p. 21. 


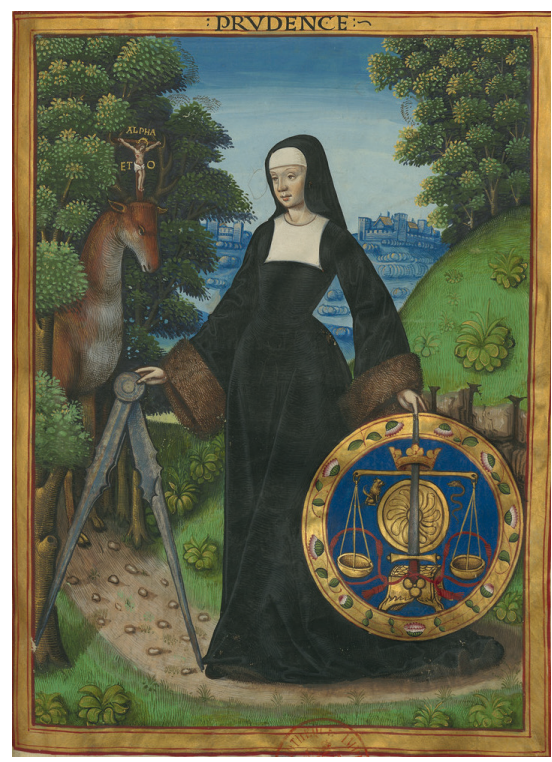

Fig. 1. François Demoulins, Retrato de Luisa de Saboya como la Prudencia, Traité des vertus cardinales, ca. 1510, París, BNF, ms. 12247.

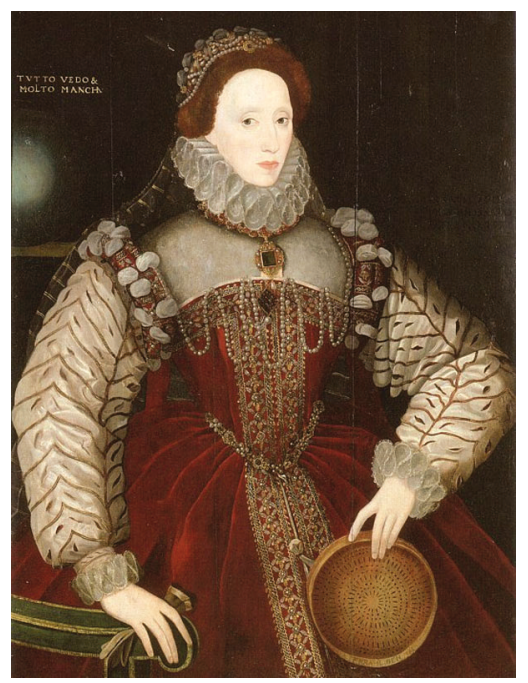

Fig. 2. Atribuido a George Gower, The Red Sieve Portrait of Queen Elizabeth I, 1579, Colección privada.

La relación directa de esta virtud con el gobernante propició la interacción visual de atributos entre la imagen de ambos, adquiriendo de esta manera la prudencia atributos propios del poder, así como el gobernante se personifica mediante atributos de dicha virtud. Francisco de Guzmán, en sus Triumphos 
morales (1587), dedicó a la prudencia Aestas non semper erit, componite nidos ['No será siempre verano / tu nido hacer procura, / mientras el verano dura']. En esta estampa se muestra un carro tirado por dragones alados y conducido por la prudencia, representada como una doncella con corona y cetro. ${ }^{56} \mathrm{La}$ prudencia también sostiene un cetro en el baldaquino de la Catedral de Santiago (Pedro del Valle, 1666-1667) y en La prudence amene la paix et l'abondance (segundo cuarto del s. XVII, París, ML) de Simon Vouet. Sin embargo, la presencia del cetro como atributo de la prudencia no es muy popular, ${ }^{57}$ sino más bien forma parte de la imagen de la justicia, con la que también comparte la peculiaridad del uso del cetro coronado con un ojo. En la Allegoria della sapienza e della prudenza (ca. 1615-1625) de Francesco Rustici la prudencia se acompaña de un cetro rematado con un ojo en el que se enrosca una serpiente. Respecto a la presencia del ojo, Gilles de Rome (1247-1316), en su De regimine principum (1277-1279) analizó la prudencia como una virtud esencialmente política, comparándola a un ojo:

La prudencia es como un ojo que permite ver el bien y el fin que se debe seguir, igual que el arquero no puede lanzar correctamente su flecha ni la dirige a su destino si no lo ve, igual que el rey no puede dirigir ni conducir a su pueblo hasta el fin que conviene si él no ve este fin gracias a su prudencia. ${ }^{58}$

Así, Gilles de Rome, concibe la prudencia como el ojo que guía por el buen camino mediante la visión de lo bueno, capacidad que dota al monarca de guiar a su pueblo por un camino de bondad. Esta reflexión se ve representada siglos después con la presencia del ojo en la imagen de la prudencia, bien como un tercer ojo o inserto en el cetro que porta. Por otro lado, Andrés Mendo representa una corona en lugar de un cetro para referirse a la prudencia que debe tener el gobernante, pero añadiendo, asimismo, la explicación sobre el cetro dotado de ojos. En su empresa Officium regis, officium capitis ['Trabajo del rey, trabajo de la cabeza'] Mendo explica:

El más noble sentido de la cabeza es la vista; y el Príncipe ha de ser todo ojos, desvelado en las convennencias de sus súbditos. [...] En Gerolyphicos [sic] ponían los Egypcios el Cetro Real con ojos; denotando, que no podía regir sin ellos a su Reyno, y qye la dicha déste consistía en tener un Príncipe prudente..$^{59}$

56. «Vestida de negro la primera / daquellas quatro damas declaradas, / bordados los vestidos a manera / de llamas por el ayre meneadas... / prudencia por Latinos es llamada: / De todas sus hermanas consegera / por ser la más de todas avisada, / sin cuya refulgente disciplina / la más prudente dellas desatina». GUZMáN: Triumphos, fol. 66r.

57. En anteriores estudios ya se ha expuesto la visualización de la prudencia, de la cual destacan como atributos principales el espejo, la serpiente y el aspecto bifaz/trifaz. No obstante, la imagen de esta virtud es muy compleja, plasmada en un tipo iconográfico sujeto a la continuidad y variación de sus atributos a lo largo del tiempo. Véase Autora: «Los fundamentos de la visualidad de la prudencia», en Imago: Revista de Emblemática y Cultura Visual, Universitat de València, Valencia, pp. 97-114.

58. Citado por Chopin-Pagotto: La prudence, p. 93.

59. AndrÉs Mendo: Príncipe perfecto y ministros aivstados, Horacio Boissat y George Remevs, Lyon, 1662, p. 47. 
Con un fin semejante, Saavedra, en su empresa Fide et diiffide, ['Confía y desconfía'], representó ojos y manos haciendo referencia a la prudencia que debe tener el gobernante antes de realizar acuerdos para no ser engañado: «Ninguna cosa mejor ni más provechosa a los mortales que la prudente difidencia. [...] El príncipe que se fiare de pocos gobernará mejor su Estado. [...] Y, cuando para su confirmación diere la mano, sea mano con ojos, primero mire bien lo que hace» ${ }^{60}$ Por su parte, Juan de Borja representa un trono en la empresa Virum ostendunt, poniendo en relación, de nuevo, la virtud de la prudencia con el acto de gobernar: «La mayor prueba, que puede a ver, para conocer el valor, y la prudencia, que un hombre tiene, es, el entregarle un cargo, ò, un Govierno». ${ }^{61}$ Sin embargo, Borja no solo emplea objetos propios del gobierno como imagen de este, ya que en la empresa Vi frangitur, obsequio flectitur ['Con fuerza se rompe; con maña, o blandura se dobla'] hace referencia a la prudencia como virtud del gobernante representando unas ramas que no se doblan:

Por lo contrario los valerosos, prudentes y bien acondicionados, les parece que seguir el medio es lo más acertado. [...] Porque de la misma manera que el que por fuerza, y sin otro término, quisiere gobernar y tratar negocios, será temido pero no amado, y siendo violento no será perpetuo su gobierno, así por lo contrario, el que con prudencia, y blandura gobernare, y tratare juntando la fuerza de hazer justicia a la blandura y maña en el modo de hacerla, sin romper, doblará a los ánimos de los que gobernare, obligándoleo a hacer por amor y respeto lo que por solo rigor no harían. ${ }^{62}$

De este modo, Juan de Borja explica mediante esta metáfora visual la necesidad de la prudencia en los actos del gobernante. Asimismo, Sambucus creó un emblema para referirse a la necesidad de los príncipes de confiar en ministros debidamente templados y prudentes, ${ }^{63}$ el cual lleva por mote Prudentibus ministris utendum ['servirse de los servidores prudentes']. Igualmente, Horapolo, en sus Hyeroglyphica (1505), representa a la prudencia en relación con el gobernante en varios jeroglíficos, como es el caso de Rey guardián, en el que hace referencia al rey vigilante como gobernante prudente. ${ }^{64}$

Sin embargo, la prudencia asociada al gobernante no siempre lleva atributos propios de la realeza, como ocurre en un arco triunfal que fue alzado en Lille en honor de Felipe II sobre el que Calvete de la Estrella describe: «la prudencia, sentada debaxo de un dosel de terciopelo carmesí, [...] tenía un compás en la

60. Diego de SaAvedra: Idea de vn príncipe político christiano, Francisco Ciprés, Valencia, 1675, p. 37.

61. JUAn DE BorJa: Empresas morales, Francisco Foppens, Bruselas, 1680, fol. 266.

62. BORJA: Empresas, p. 22.

63. Johannes Sambucus: Emblemata, cum aliquot numis antiqui operis, Ioannis Sambuci Tirnaviensis Pannonii, Amberes, 1564, p. 238.

64. Horapolo: Hyerogliphica, Akal, Madrid, 1991, p. 197. 
mano y en medio del architrabe esta letra: PrVDentia Vestra gVbernatvr Orbis terrarvm. Por vuestra prudencia es gobernado el mundo». ${ }^{65}$ Así es como la prudencia se alza como principal virtud del gobernante, representándose incluso a su lado, como su fiel consejera y guía: «Estava la prudencia al lado derecho del Príncipe, que era una doncella ricamente vestida y decía: Me DVCe tVtVs Coelvm fama RebvsQve gestis AeQVabis. Siendo yo vuestra guía, seguro llegaréys al cielo con gran fama y hechos esclarecidos». ${ }^{66}$ Ante dicha relación tuvo lugar la interacción de atributos entre la imagen del gobernante y la de la prudencia. La serpiente, bautizada como prudente según las palabras de san Mateo, ${ }^{67}$ personifica al propio gobernante en el jeroglífico de Horapolo que tiene por lema Rey muy poderoso. En este jeroglífico se representa una serpiente enroscada con el nombre del rey (Apis) entre la espiral que forma su cuerpo, imagen casi idéntica a la que Jean Baudoin presenta bajo el lema Des mauvais conseils; et que ceux qui les donnent s'en trouvent $m a l^{68}$ (fig. 3). De este modo, la serpiente se expresa como imagen del rey que domina el mundo desde su prudencia, haciendo referencia a esta como virtud del gobernante ${ }^{69}$ como bien muestra una estampa de la obra de Marciano Marcello Pompe fvnebri dell'vniverso: nella morte di Filippo Qvarto il grande re delle Spagne. ${ }^{70}$ También en la empresa 28 de Diego de Saavedra, Quae sint, quae fuerint, quae mox ventura trahantur ['Las que son, las que fueron, las que traerá la fortuna'], ${ }^{71}$ presenta una pictura en la que la el gobernante prudente se visualiza tal y como se expone:

Todos estos tiempos significa esta empresa en la serpiente, símbolo de la prudencia, revuelta al ceptro sobre el reloj de arena, que es el tiempo presente que corre, mirándose en los dos espejos del tiempo pasado y del futuro... Todos tres tiempos son espejo del gobierno, donde, notando las manchas y defectos pasados y presentes, se pule y hermosea, ayudándose de las experiencias propias y adquiridas. $^{72}$

65. Juan Cristóbal Calvete de la Estrella: El felicíssimo viaje del muy alto y muy poderoso príncipe Don Phelippe, Sociedad Estatal para la Conmemoración de los Centenarios de Felipe II y Carlos V, Madrid, 2001, p. 247.

66. Calvete de la Estrella: El felicísimo, p. 254.

67. «Sed, pues, prudentes como las serpientes, y sencillos como las palomas» (Mateo,10,16). La prudencia es cautelosa como la serpiente ya que, como ella, se desliza sobre la tierra sin hacer ruido, así como va con precaución sobre el terreno dificultoso, como bien expone la patrística al comentar el evangelio de san Mateo. Véase TerT. adv. Val. 2 (PL 2,544); Avg. doctr. christ. 3,25 (pl 34,79); Avg. serm. 64,1-2 (PL 38,425426); Greg. M. epist. 13,40 (PL 77,765); Hier. in psalm. 83 (pl 26,1074).

68. Jean Baudoin: Recueil d'emblêmes, Jean Baptiste Loyson, París, 1659, vol. 2, p. 59.

69. Horapolo: Hyerogliphica, p. 193.

70. His dvcibvs princeps celebraberis ore virorv illa homines beat haec sideribvs sq locat, Antonio Tempesta, 1590

71. Saavedra toma la misma fuente que Sebastián de Covarrubias en el emblema (VIRG. Gramm., Georgicas, 4, 393) en el emblema que lleva el mismo título, aunque ambos autores lo visualizan de distinto modo.

72. SAAVEDRA: Idea de vn príncipe, p. 172. 


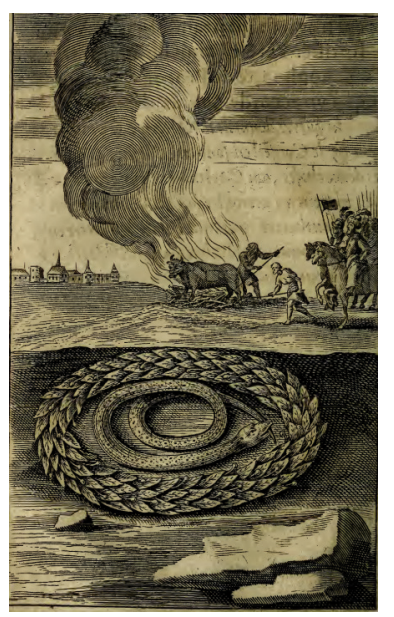

Fig. 3. Jean Baudoin, «Des mauvais conseils; et que ceux qui les donnent s'en trouvent mal», Recueil d'emblêmes, 1685.

La empresa alude a la educación del futuro rey en la virtud de la prudencia, ${ }^{73}$ haciéndole reflexionar sobre el paso del tiempo, así como destacando que para actuar prudentemente en el presente debe valorar lo pasado y las consecuencias que puede traer el futuro, razón por la cual también aparece un reloj de arena. ${ }^{74}$ Casi idéntica es la imagen que presenta Jean Baudoin en Des princes en general et des qualitez qui les rendent considerables (1685), ${ }^{75}$ donde, aunque sin serpiente, un cetro se sitúa entre dos espejos que muestran su reflejo (fig. 4). Baudoin explica que el cetro es el príncipe, como en el emblema de Saavedra, quien tiene que tener en cuenta el pasado para obrar bien en el futuro y, por lo tanto, dejarse guiar por la prudencia. ${ }^{76}$ Pero no es el único emblema que Baudoin dedicó a dicha relación. Bajo el mote De la necessite du Conseil, et que la Prudence y doit estre jointe (fig. 5), Baudoin representó a la prudencia sosteniendo un cetro y una espada en la que se enrosca una serpiente, explicando al respecto: «Cette Reyne qui tient des deux mains vn Sceptre \& vne Espée, où est enlace vn Serpent couronné, nous enseigne que le Conseil, \& la Prudence produisent de merueilleux effets, quan l'vn \& l'autre agissent ensemble». ${ }^{77}$ Así, bajo la práctica de la prudencia y el consejo tendrá lugar un buen gobierno.

73. Luís Vives FerRándiz: Vanitas: retórica visual de la mirada, Encuentro, Madrid, 2011, pp. 95-96.

74. Véase María del Carmen García: «El símbolo del espejo en la obra de Saavedra Fajardo, Idea de un príncipe político christiano representada en cien emblemas», en José Miguel Morales, Reyes EsCALera y Francisco J. Talavera (coors.): Confluencia de la imagen y la palabra, Universitat de València, València, 2015, p. 230.

75. BAudoin: Recueil d'emblêmes, vol. 1, p. 283.

76. Los estados del tiempo son una de las características esenciales en la práctica de la prudencia, lo que se representa en la imagen de esta virtud de diversos modos, como ya se ha estudiado. Véase AUTORA: «El tiempo en la visualidad de la prudencia», en Blanca Ballester, Antonio Bernat i John J. Cull (eds.): Encrucijada de la palabra y la imagen simbólicas, José J. de Olañeta, Palma de Mallorca, 2017, pp. 505-516.

77. BAudoin: Recueil d'emblêmes, vol. 2, p. 291. 


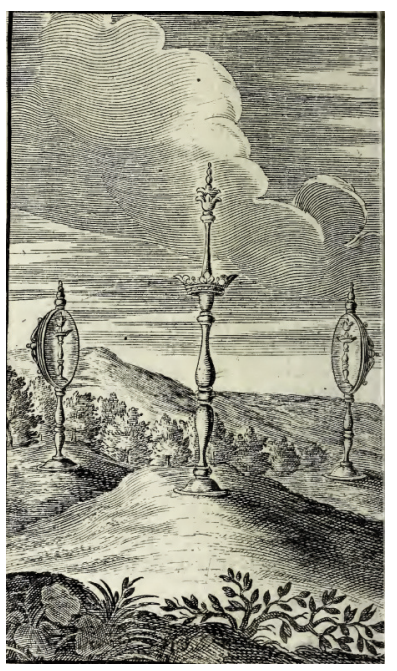

Fig. 4. JEAN BAUdoin, «Des princes en general et des qualitez qui les rendent considerables», Recueil d'emblêmes, 1685.

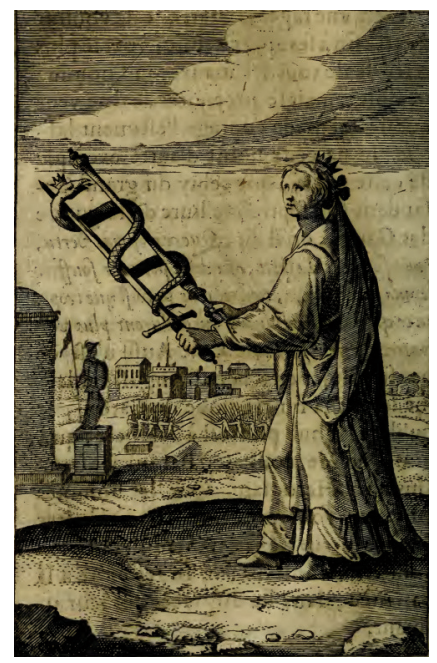

Fig. 5. Jean Baudoin, «De la necessite du Conseil, et que la Prudence y doit estre jointe», Recueil d'emblêmes, 1685.

Desde Tirso de Molina, «Del cielo santo imagino / de mi madre la prudencia / con que el reino gobernó», ${ }^{78}$ hasta John Austin con su poema Hymn (1668), «PRUDENCE governed all the rest; / Prudence made us still apply / What was fittest, what was best / To advance great CHARITY» ${ }^{79}$ son inconta-

78. Tirso de Molina: La prudencia en la mujer, p. 54, en: www.librodot.com (consultado el 15/01/2019).

79. Citado por Giles Fletcher: Christ's Victory and Triumph in Heaven and Earth Over and After Death, E. P. Dutton, Nueva York, 1888, p. 181. 
bles los escritores y obras que tratan la prudencia como la virtud esencial para todo gobierno. Muestra de ello es Fray Luis de Granada, quien explicó dicha relación describiendo imágenes que más tarde se asociarán a la prudencia del gobernante:

Esta virtud, en la vida espiritual, es lo que los ojos al cuerpo, lo que el piloto en el navío, lo que el rey en el reino y lo que el gobernador en el carro, que tiene por oficio llevar las riendas en la mano y guiarlo por donde ha de caminar. Sin esta virtud, la vida espiritual sería toda ciega, desproveída (sic.), desconcertada y llena de confusión. ${ }^{80}$

Por este motivo, en ocasiones la presencia de un timón hace referencia a la prudencia. El conde-duque de Olivares, valido de Felipe IV, se hizo retratar por Rubens rodeado de diferentes alegorías (grabado de Paulus Pontius, 1626, Madrid, MP), entre las cuales aparecen el timón y la bengala, que quieren significar la prudencia del gobernante. ${ }^{81}$ Según Barbier, ${ }^{82}$ la prudencia se representa con un timón para guiarse entre los peligros, lo que podemos ver en una escultura de Hans Krumper (1614-1616, Múnich, Residenz) o en la alegoría de la prudencia que acompaña el Retrato de Gustaaf Willem, barón de Imhoff (Jan Caspar Philips y Hermanus Besseling, 1742, Ámsterdam, RijM, RP-P-OB-60.084). En el Sepulcro del cardenal Mazarino (Antoine Coysevox, 1692, París, ML), la prudencia, además de sostener un timón en su mano derecha, reposa sus pies sobre un globo terráqueo, lo que muestra la necesidad de esta virtud para gobernar el mundo, como también vemos en la Alegoría de la Felicidad de Agnolo Bronzino (1564, Florencia, GU) lo representan junto a dicha virtud.

\section{Prudencia militar}

Los antecedentes mitológicos de la prudencia, encarnados en la imagen de la diosa Atenea/Minerva -que simboliza la guerra justa y prudente- ${ }^{83}$ influyeron posteriormente en la representación de esta virtud armada o portando atributos propios de un guerrero. Al respecto, santo Tomás de Aquino, dentro de las partes esenciales o especies de la prudencia en lo que respecta al Estado y al bien común distingue la "prudencia militar», consideración que basa en las fuentes bíblicas:

80. Luís de Granada: Guía de pecadores, Administración del Apostolado de la Prensa, Madrid, 1906, vol. 1, p. 424.

81. Carmona: Iconografía, p. 232.

82. Xavier Barbier: Orné de 39 planches comprenant 394 dessins par M. Henri Nodet, Societé de libraire ecclésiastique et religeuse, París, 1898, p. 213.

83. María Cruz Martínez: Mitología clásica e iconografía cristiana, Editorial Universitaria Ramón Areces, Madrid, 2010, p. 67. 
Está lo que leemos en la Escritura: Con estratagemas se hace la guerra, y la victoria está en la muchedumbre de los consejos (Pr 24,6). Pues bien, aconsejar es propio de la prudencia. Luego en asuntos de guerra es sumamente necesaria la especie de prudencia llamada militar. ${ }^{84}$

Más tarde, Cristina de Pizán, en su Epitre d'Othea, volvió a los antecedentes mitológicos de la prudencia para explicar su vertiente militar: «Minerva fue una dama muy sabia que inventó el arte de forjar armaduras, cuando, antiguamente, la gente no podía armarse más que con cuero hervido, y por su gran prudencia, la hicieron diosa». ${ }^{85}$ Por un lado, Platón ya entendió la prudencia como una parte de la sabiduría y, por otro, los griegos y romanos concebían a Atenea/Minerva como representación del «pensamiento armado». ${ }^{86}$ Por otra parte, la imagen armada de una virtud corresponde más bien a la fortaleza, lo que santo Tomás también explica: «El ejercicio del arte militar corresponde a la fortaleza; la dirección, empero, a la prudencia, sobre todo tal como se ve en el jefe del ejército». ${ }^{87}$ En todo momento cabe recordar que las virtudes cardinales son conceptos dependientes unos de otros en cuanto a que completan las funciones y actos de las otras, en este caso la prudencia en su vertiente militar completa el ejercicio de la fortaleza. Con este fin, Juan Francisco Villava representa en la empresa que lleva por lema Hoc insuper addo ['Además, añado esto'] un salero, que quiere significar:

Sacrificio sin sal no se admitía / para que entienda el justo, / que aunque en heroicas obras para el cielo, / con abrasado zelo / se ocupe noche y día, / no puede dar a Dios perfecto gusto, / si prudencia le falta. / Porque es virtud tan alta, / que como sal, en todas derramada / les da el sabor, que más a Dios le agrada. ${ }^{88}$

De este modo, Villava ensalza la primacía de la prudencia frente a las otras virtudes a la vez que muestra su dependencia entre ellas: «Porque si la virtud consiste en el medio, y esta elección a de hazer la prudencia, la sal de las virtudes la prudencia es, que las sala, las mide y conserva, para que no se corrompan». ${ }^{89}$ Sin embargo, a pesar de la dependencia entre las virtudes, la esencia de todas ellas es la prudencia: «Por la Sal cosa es común significarse la prudencia, la qual es la suprema de la Virtudes morales, y la que abraça todas $[. .$.$] donde no ay prudencia, no ay virtud» .^{90}$ A partir de estas premisas,

84. S. Th. [41154] IIa-IIae q. 50 a. 4 s. c.

85. PizÁN: La rosa y el príncipe, p. 103.

86. Jean-Christian Tautil: «Prudence, Force et Tempérance dans le décor de la Chambre de la Signature», en Luigi De Poli (coor.): La Représentation de la Prudence, col. Chroniques Italiennes, Université de la Sorbonne Nouvelle-París III, París, 1999, p. 110.

87. S.Th. [41158] II ${ }^{\mathrm{a}}$-IIae q. 50 a. 4 ad 3.

88. JuAn Francisco de Villava: Empresas espirituales y morales, Fernando Díaz de Montoya, Baeza, 1613, parte I, empresa 19, fol. 51r.

89. Villava: Empresas espirituales, fol. 51r.

90. Ibid. 
Rafael representa la prudencia en la Stanza di Constantino de los Palacios Vaticanos como una «Minerva cristiana $»^{91}$ que viste yelmo y armadura. Ripa, en su Iconología, concibe la prudencia vistiendo un yelmo: «Mujer con yelmo de color dorado que la cabeza le cubre». ${ }^{92}$ Según Barbier, ${ }^{93}$ esta viste un yelmo con el fin de proteger su cabeza y defenderse contra toda sorpresa, por lo que así se representa en una estampa acompañando al príncipe de Orange (Gerard de Lairesse y Johannes Jacobsz van den Aveele, 1672, Ámsterdam, RijM, RP-P-OB-67.810), completando su imagen con el aspecto bifaz, el espejo y la serpiente enroscada a una lanza. También se muestra la prudencia con yelmo y espejo en la estampa Resultado del pacto federal del memorable día del 14 de julio de 1790 (Jean Henri Alexandre Pernet, 1790, BNF, París, RESERVE FOL-QB-201 (122)), donde aparece junto a Luis XVI, y en una pintura de Franz Georg Hermann (s. XVIII, Stöttwang, San Gordian y San Epimachus). En ocasiones, el yelmo representa a la prudencia sin encontrarse esta personificada, constituyendo el atributo como una alegoría en sí de esta virtud, lo que se complementa con el aspecto bifaz en la estampa de El comandante Orange comparado con Josué (1569) de Mathis Zündt (fig. 6).

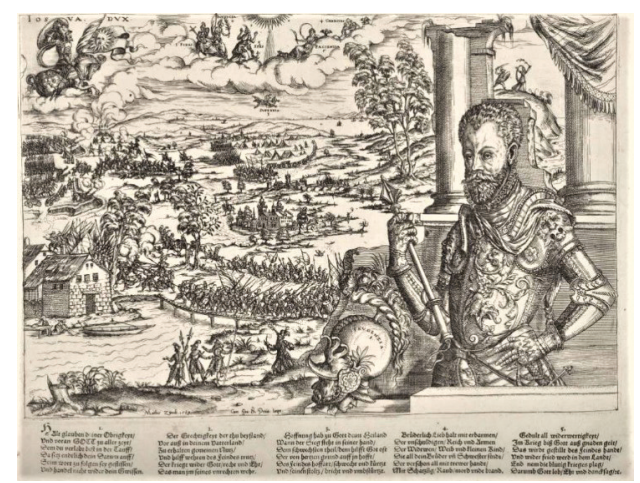

Fig. 6. Mathis ZüNDT, El comandante Orange comparado con Josué, 1569, Ámsterdam, RijM, RP-P-OB-79.064.

Igualmente, encontramos casos del mismo gobernante representado como la prudencia portando los atributos que la identifican, como Felipe de Macedonia como la prudencia (Tommaso di Andrea Vincidor, ca. 1526-1538, Breda, Onze Lieve Vrouwekerk \& Grote Kerk, nördliches Seitenschiff).

\footnotetext{
91. TAutil: Prudence, Force, p. 110.

92. Ripa: Iconología, vol. 2, p. 234.

93. BARbier: Orné de 39 planches, p. 212.
} 


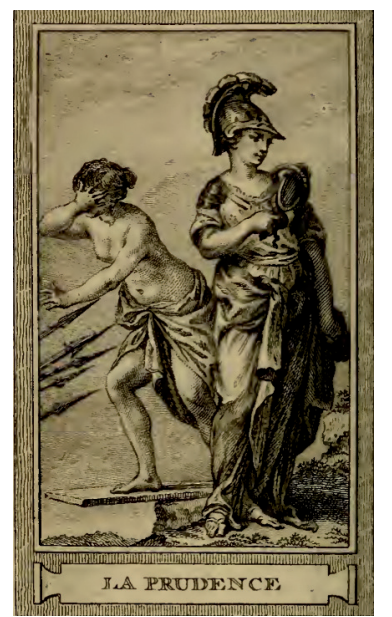

Fig. 7. H. F. Gravelot, C. N. Cochin y C. E. Gaucher, La Prudence, Iconologie par figures..., 1791.

También Gravelot al representar a la prudencia con aspecto guerrero (fig. 7) explica «Quelquefois aussi l'one donne à la Prudence un casque d'or, ce qui signifie que l'homme prudent siat résister aux embûches de la fraude et de la perfidie». ${ }^{94}$ De este modo, el aspecto guerrero de esta virtud se justifica mediante la significación de sus atributos. Asimismo, Jean Baudoin, bajo el mote Du Conseil des Princes ${ }^{95}$ representa a Atenea como alegoría misma del consejo y la prudencia que necesitan los príncipes para gobernar. ${ }^{96}$ Respecto al yelmo, Morales $^{97}$ explica que significa que el hombre prudente sabe resistir los embates del fraude y de la perfidia, los cuales también suelen representarse en la imagen de la prudencia mediante la máscara. ${ }^{98}$ Quizás por este motivo, en una de las estampas de los Triumphos morales (fol. 176v) de Francisco de Guzmán es la propia prudencia quien viste a Felipe II con un yelmo (fig. 8), ${ }^{99}$ mientras las otras virtudes cardinales le proporcionan otras prendas. Sin embargo, Guzmán

94. Hubert F. Gravelot, Charles N. Cochin y Charles-Etienne Gaucher: Iconologie par figures, Lattré Graveur, París, 1791, vol. 4, p. 43.

95. Baudoin: Recueil d'emblêmes, vol. 2, p. 137.

96. «L'ancienne Fable nous fais acoriure, que Iupiter ayant aspousé Metis, c'est à dire le temps de là qu'elle estoit enceinte; ce qui fut cause qu'il la deuora tout aussi-tost; sans vouloir attendre qu'elle accouchast; si bien que luy mesme deuint gros, \& enfanta Pallas, qui par vn merueilleux effet nasquit de son cerueau tout armée». BAudoin: Recueil d'emblêmes, vol. 2, p. 137.

97. José Luís Morales: Diccionario de términos artísticos, Edelvives, Zaragoza, 1985, p. 281.

98. Cabe tener en cuenta que las máscaras y anteojos eran usados a finales del siglo xvi para denotar la mentira y el engaño. JoHn B. KNIPPING: Iconography of the Counter Reformation in the Netherlands: Heaven on earth, B. de Graaf, Nieuwkoop, 1974, p. 20. La máscara constituye un elocuente símbolo del vicio, por lo que Ripa concede este atributo al engaño, la mentira y al ocio. Consecuentemente, su aparición bajo los pies de esta virtud significa el triunfo moral de esta sobre aquel, constituyendo una psicomaquia.

99. GuZmán: Triumphos, fols. $177 \mathrm{r}$ y $179 \mathrm{v}$ 
no fue el primero en representar a las virtudes cardinales vistiendo a un gobernante, puesto que ya encontramos esa imagen en diferentes manuscritos de Champion des Dames (1488, París, BNF, Rés. Ye-27; y 1440, BNF, Français 12476, fol. 8v) de Martin Le Franc.

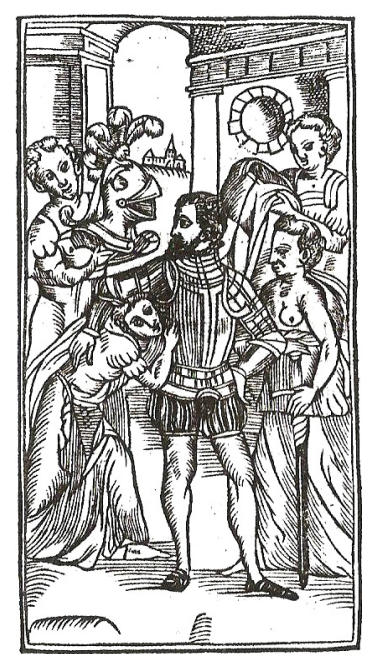

Fig. 8. Francisco DE GuZmán, «Las virtudes y la fama del Rey Felipe II», 1565, Triumphos morales.

Por otro lado, en la estampa Felipe III rodeado de los emblemas del buen gobierno de la obra de Juan Antonio de Vera y Zúniga El enbaxador (1620, Sevilla), el monarca aparece rodeado por una serie de emblemas relacionados con sus virtudes. Dos figuras proclaman su fama en los dos hemisferios y flaquean una fuente en forma de concha con el lema Nec Satis. El rey está representado como guerrero, con armadura, pero su fuerza se apoya, de un lado, en el conocimiento de la doctrina política y de la historia y, de otro, en la prudencia, plasmadas en los tres libros que sirven de base al casco: uno de Philippe de Commines, posiblemente Les memoires, el Gobernador cristiano de J. Márquez y la Política cristiana. ${ }^{100}$ En esta imagen guerrera del rey que se asocia a las virtudes como poseedor de las mismas, vemos a esa Minerva armada que representa la guerra desde la sabiduría y la prudencia que la caracterizan, lo que se traduce en esa «prudencia militar» que Santo Tomás le atribuye a cualquier gobernante.

100. Biblioteca Nacional (España). Servicio de Dibujos y Grabados, op. cit., p. 205. 


\section{Conclusiones}

Por lo tanto, todas estas apariciones de la prudencia en las entradas del monarca, en sus retratos o en sus monumentos funerarios, trataban de representar las virtudes y valores que tanto se esgrimían en la educación del príncipe y de rechazar aquellos que contravenían dicha educación. De este modo, la actuación del monarca se erigía como modelo de referencia, ilustrando el planteamiento de la nueva prudencia, convirtiéndose en un nuevo paradigma con su virtualidad educativa, ${ }^{101}$ pues así explica Antonio de Herrera y Tordesillas la asociación entre «monarca» $\mathrm{y}$ «prudencia»: «Y assi acabo este gran Monarca con la misma prudencia con que vivio: por lo qual [méritamente] se le dio el atributo de Prudente». ${ }^{102}$ Consecuentemente, la vasta presencia de representaciones de la prudencia junto al gobernante, así como la asociación de la virtud a este, denota la importancia de la posesión de dicha virtud por el monarca, como buen gobernante y como buen cristiano. Para ello se muestra junto a él y en continua asociación a sí mismo para recordar lo ideal de su persona para ejercer su cargo a nivel civil y religioso.

\section{Bibliografía}

Alfonso, Rey de Castilla: Las siete partidas del Rey Don Alfonso el Sabio. Benito Monfort, Valencia, 1767, fol. 4v. Disponible en Biblioteca Virtual de Pensamiento Político Hispánico Saavedra Fajardo. Partida II, Título V, Ley VIII.

Baudoin, Jean: Recueil d'emblêmes, Jean Baptiste Loyson, París, 1659.

Barbier, Xavier: Orné de 39 planches comprenant 394 dessins par M. Henri Nodet, Societé de libraire ecclésiastique et religeuse, París, 1898.

Bermudo, José Manuel: Maquiavelo, consejero de príncipes, Universitat de Barcelona, Barcelona, 1994.

BORJA, JUAN DE: Empresas morales, Francisco Foppens, Bruselas, 1680.

CAlvete de la Estrella, JuAn CRistóbal: El felicíssimo viaje del muy alto y muy poderoso príncipe Don Phelippe, Sociedad Estatal para la Conmemoración de los Centenarios de Felipe II y Carlos V, Madrid, 2001.

Carmona Muela, Juan: Iconografía clásica: guía básica para estudiantes, Istmo, Madrid, 2002.

Checa Cremades, Fernando: Carlos Vy la imagen del héroe del Renacimiento, Taurus, Madrid, 1987.

Chew, Samuel C.: The virtues reconciled: an iconographic study, University of Toronto Press, Toronto, 1947.

Chopin-Pagotto, Myriam: «La prudence dans les Miroirs du prince», en Luigi de Poli (coor.): La Représentation de la Prudence, col. Chroniques Italiennes, Université de la Sorbonne Nouvelle-París III, París, 1999, pp. 87-98.

101. Fernández Albaladejo: Espejo de prudència, pp. 73-74.

102. Citado por Fernández Albaladejo: Espejo de prudència, p. 70 
Dauvois, Nathalie: «Prudence et politique chez les grands rhétoriqueurs: Janus Bifrons», en Evelyne Berriot-Salvatore, Catherine Pascal y François Roudaut (dir.), La vertu de prudence: entre Moyen Âge et âge classique, Classiques Garnier, París, 2012, pp. 55-71.

Fernández Albaladejo, Pablo: «Espejo de prudencia», en Francisco J. Rocha (coor.): La monarquía hispánica Felipe II, un monarca y su época: Real Monasterio de San Lorenzo del Escorial, 1 de junio, 10 de octubre, 1998, Sociedad Estatal para la Conmemoración de los Centenarios de Felipe II y Carlos V, Madrid, 1998, pp. 91-187.

Ferro, Jorge Norberto: «Las virtudes del gobernante en las cuatro crónicas que preceden a la obra del canciller Ayala», en Anales de la Fundación Francisco Elías de Tejada, Fundación Francisco Elías de Tejada, Madrid, 1995, 1, pp. 49-61.

Fletcher, Giles: Christ's Victory and Triumph in Heaven and Earth Over and After Death, E. P. Dutton, Nueva York, 1888.

García Estradé, María del Carmen: «El símbolo del espejo en la obra de Saavedra Fajardo, Idea de un príncipe político christiano representada en cien emblemas», en José MigueL Morales, Reyes Escalera y Francisco J. Talavera (coor.): Confluencia de la imagen y la palabra, Universitat de València, València, 2015, pp. 225-240.

Giamboni, Bono: Il libro de' vizî e delle virtudi e Il trattato di virtú e di vizî, G. Einaudi, Turín, 1968.

Granada, Luís De: Guía de pecadores, Administración del Apostolado de la Prensa, Madrid, 1906, vol. 1.

Gravelot, Hubert F.; Cochin, Charles N. y Gaucher, Charles-Etienne: Iconologie par figures, Lattré Graveur, París, 1791, vol. 4.

GuZMán, Francisco de: Triumphos morales, Casa de Andrés, Alcalá de Henares, 1565.

Horapolo: Hyerogliphica, Akal, Madrid, 1991, p. 197.

IMPERIAL, FRANCISCO DE: El dezir a las syete virtudes y otros poemas, Espasa-Calpe, Madrid, 1977, pp. 107-112.

KLeine, MARina: «La virtud de la prudencia y la sabiduría regia en el pensamiento político de Alfonso X el Sabio», en Res Publica: Revista de Filosofía Política, Universidad de Murcia, Murcia, 2007, pp. 223-240.

KNipping, John B.: Iconography of the Counter Reformation in the Netherlands: Heaven on earth, B. de Graaf, Nieuwkoop, 1974.

LAURent, Frère: Le somme le roi, Paillarr, París, 2007.

Lehmann, Yves: «Prudentia chez les penseurs romains. Essai d'investigation philosophique et morale», en Luigi de Poli (coor.): La Représentation de la Prudence, col. Chroniques Italiennes, Université de la Sorbonne Nouvelle-París III, París, 1999, pp. 13-19.

Maquiavelo, Nicolás: El príncipe, Edaf, Madrid, 2009.

—: El príncipe (Comentado por Napoleón Bonaparte), Santiago Rueda, Buenos Aires, 1968.

Martínez de la Torre, María Cruz: Mitología clásica e iconografía cristiana, Editorial Universitaria Ramón Areces, Madrid, 2010.

MenA, JUAN DE: «Coplas que fizo el famoso Juan de Mena contra los pecados mortales», en Raymond Foulché-Delbosch: Cancionero castellano del siglo XV, Bailly Bailliére, Madrid, 1912.

Mendo, Andrés: Príncipe perfecto y ministros aivstados, Horacio Boissat y George Remevs, Lyon, 1662.

MendozA, Fray ÍñIGO DE: «Vita Christi techo por coplas por frey Yñigo de Mendoca a petiçion de la muy virtuosa señora doña Juana de Cartagena», en RaYmond Foulché-Delbosch: Cancionero castellano del siglo XV, Bailly Bailliére, Madrid, 1912.

Molina, Tirso DE: La prudencia en la mujer, p. 54, en: www.librodot.com (consultado el 15/01/2019).

Muñoz Gómez, Elena: «El tiempo en la visualidad de la prudencia», en Blanca Ballester Morell, Antonio Bernat Vistarini i John J. Cull (eds.): Encrucijada de la palabra y la imagen simbólicas, José J. de Olañeta, Palma de Mallorca, 2017, pp. 505-516. 
-: «Los fundamentos de la visualidad de la prudencia», Imago: revista de emblemática y cultura visual, Universitat de València, Valencia, pp. 97-114.

Morales, José Luís: Diccionario de términos artísticos, Edelvives, Zaragoza, 1985.

PizÁN, CRISTINA DE: La rosa y el príncipe: voz poética y voz política en las „Epístolas“, Gredos, Madrid, 2005.

Richter Sherman, Claire: Imaging Aristotle: verbal and visual representation in fourteenthcentury France, University of California Press, Berkeley, 1995.

RipA, CesAre: Iconología, Akal, Madrid, 2007, vol. 2.

RUCQUOI, AdELINE: «De los reyes que no son taumaturgos: los fundamentos de la realeza en España», en Temas medievales, Programa de Investigaciones Medievales, Consejo Nacional de Investigaciones Científicas y Técnicas, Buenos Aires, 1995, p. 163-186.

SaAvedra Fajardo, Diego de: Idea de vn príncipe politico christiano, Francisco Ciprés, Valencia, 1675.

Sambucus, Johannes: Emblemata, cum aliquot numis antiqui operis, Ioannis Sambuci Tirnaviensis Pannonii, Amberes, 1564.

Tautil, Jean-Christian: «Prudence, Force et Tempérance dans le décor de la Chambre de la Signature», en Luigi DE Poli (coor.): La Représentation de la Prudence, col. Chroniques Italiennes, Université de la Sorbonne Nouvelle-París III, París, 1999, p. 99-115.

Toste, Marco: «Virtue and the City: The Virtues of the Ruler and the Citizen in the Medieval Reception of Aristotle's Politics», en István P. BejCzy y CARy J. Nederman (eds.), Princely virtues in the Middle Ages, 1200-1500, Brepols, Turnhout, Abingdon, 2007, pp. 73-99.

Verweij, Michiel: «Princely Virtues or Virtues for Princes?». en IstVán P. BejCzy y Cary J. Nederman (eds.), Princely virtues in the Middle Ages, 1200-1500, Brepols, Turnhout, Abingdon, 2007, pp. 51-73.

VÉLEZ-SAINZ, Julio: «La iconización de lo femenino en la Edad Media (de Prudencio a la corte de Juan II)», en Tejuelo: Didáctica de la Lengua y la Literatura, 2008, pp. 57-76.

Villava, Juan Francisco de: Empresas espirituales y morales, Fernando Díaz de Montoya, Baeza, 1613.

VIVEs-FerRÁndiz, Luís: Vanitas: retórica visual de la mirada, Encuentro, Madrid, 2011.

Vv. AA.: La fiesta en la Europa de Carlos V: [exposición] Real Alcázar, Sevilla, 19 de septiembre-26 de noviembre 2000, Sociedad Estatal para la Conmemoración de los Centenarios de Felipe II y Carlos V, Madrid, 2000. 